\title{
Reading in a Regular Orthography: An fMRI Study Investigating the Role of Visual Familiarity
}

\author{
Anja Ischebeck ${ }^{1}$, Peter Indefrey ${ }^{2,3}$, Nobuo Usui ${ }^{4}$, Izuru Nose ${ }^{5}$, \\ Frauke Hellwig ${ }^{2,3}$, and Masato Taira ${ }^{4}$
}

\begin{abstract}
In order to separate the cognitive processes associated with phonological encoding and the use of a visual word form lexicon in reading, it is desirable to compare the processing of words presented in a visually familiar form with words in a visually unfamiliar form. Japanese Kana orthography offers this possibility. Two phonologically equivalent but visually dissimilar syllabaries allow the writing of, for example, foreign loanwords in two ways, only one of which is visually familiar. Familiarly written words, unfamiliarly written words, and pseudowords were presented in both Kana syllabaries (yielding six conditions in total) to participants during an fMRI measurement with a silent articulation task (Experiment 1) and a phonological lexical decision task (Experiment 2) using an event-related design. Consistent over two experimental tasks,
\end{abstract}

\section{INTRODUCTION}

Models of normal reading often assume two different pathways to arrive at the correct pronunciation of a written word. In the dual-route model of reading (for a review, see Coltheart, Curtis, Atkins, \& Haller, 1993) it is assumed that the pronunciation of a word can be either assembled sequentially from the word's letters or graphemes according to a set of grapheme to phoneme conversion rules (GPC rules) or retrieved directly from memory if the visual form of the word as a whole has been recognized. For example, pseudowords (pronounceable nonwords) can only be read by phonological assembly according to the GPC rules, whereas words with an irregular spelling, that is, a spelling that does not conform to the GPC rules of the respective orthography, are read more successfully via the visual word form lexicon. Orthographically regular written words on the other hand can be read via both routes.

A number of studies have ventured to identify the neural correlates of these two routes but yielded diver-

\footnotetext{
${ }^{1}$ University of Nijmegen, ${ }^{2}$ Max Planck Institute for Psycholinguistics, ${ }^{3}$ F. C. Donders Center for Cognitive Neuroimaging, ${ }^{4}$ Nihon University Graduate School of Medical Science, ${ }^{5}$ Bunkyo Gakuin University
}

the three different stimulus types (familiar, unfamiliar, and pseudoword) were found to activate selectively different brain regions previously associated with phonological encoding and word retrieval or meaning. Compatible with the predictions of the dual-route model for reading, pseudowords and visually unfamiliar words, which have to be read using phonological assembly, caused an increase in brain activity in left inferior frontal regions (BA 44/47), as compared to visually familiar words. Visually familiar and unfamiliar words were found to activate a range of areas associated with lexico-semantic processing more strongly than pseudowords, such as the left and right temporo-parietal region (BA 39/40), a region in the left middle/inferior temporal gyrus (BA 20/21), and the posterior cingulate (BA 31).

gent results. The route for phonological encoding using the GPC rules, also referred to as assembled phonology, has been identified with the left inferior frontal region (BA 44/45) (e.g., Fiez, Balota, Raichle, \& Petersen, 1999). Other researchers have suggested that the sublexical procedure is localized in the left posterior superior temporal region (e.g., Paulesu et al., 2000). The direct route of visual word form retrieval, often referred to as accessed phonology, has been identified with a number of diverse brain regions: the left posterior middle temporal gyrus, including the angular gyrus (e.g., Howard et al., 1992), areas in the left temporo-occipital lobe, especially the left fusiform gyrus (e.g., Cohen et al., 2000), and the left and right extrastriate cortices (e.g., Petersen, Fox, Posner, Mintun, \& Raichle, 1988).

This lack of agreement might be attributable to a number of reasons, methodological as well as inherent. Methodologically, the incommensurability of the tasks and stimuli used in many subtraction designs in brain imaging research, as well as the assumptions of linearity and pure insertion, might critically impair the reproducibility of results across experiments and their interpretation (see, for a review, Friston et al., 1996). Furthermore, block designs, mandatory for PET experiments, can induce strategic effects (d'Esposito, Zarahn, \& Aguirre, 1999). Inherent reasons related to the nature of 
the neural implementation of the two routes might also account for the difficulty in finding unambiguous evidence for them. First, the cognitive functions under scrutiny might be subserved by several functionally specialized areas, highly sensitive to differences in stimulus materials and task requirements. Alternatively, visual word form processing might be so effortless that it remains inconspicuous given the signal-to-noise ratio of present imaging methods.

The present study avoids some of the methodological problems by directly comparing different script variants of the same word and pseudoword stimuli in a silent articulation task (Experiment 1). The randomization of different stimulus types in an event-related design prevents confounds due to low-frequency signal shifts (Donaldson \& Buckner, 2001) and strategic adaptation by participants. Additionally, the same materials were presented in a phonological lexical decision task (Experiment 2). In this variant of a lexical decision task, participants have to press one button if a letter string sounds like a real word, and another button if not. This task basically requires the same processing steps as the silent articulation task (see the Discussion section for a task analysis). It is thus possible to check the consistency of the results over tasks and experiments and to attribute differences in brain activation patterns to differences in the stimulus materials.

The two pathways postulated by the dual-route model can be investigated by comparing stimuli that can be read via one pathway to stimuli that can be read over the other or both pathways. Orthographically regular and visually highly familiar words (i.e., words well known to the reader) are assumed to be read over both routes, whereas the same words presented in a visually unfamiliar way can only be read using sequential phonological encoding. A comparison of both could shed some light on the neural basis of the dual-route model. There are possibilities to achieve this experimental variation in alphabetic scripts, such as mirror writing and case alternation (e.g., nIgHt). These manipulations have already been employed in neuroimaging studies (mirror writing: Dong et al., 2000; case alternation: Polk \& Farah, 2002). One limitation to this approach, however, is that these manipulations render the sublexical units of the word, letters, and graphemes unfamiliar as well. This is an obstacle for grapheme to phoneme conversion, when a grapheme consists of more than one letter or when conversion rules apply to chunks of graphemes. Observed brain activation differences might therefore be due to an increased difficulty of GPC processing rather than only to the disruption of visual word form processing.

Japanese Kana orthography, on the other hand, allows a more direct realization of this manipulation without simultaneously rendering sublexical units unfamiliar as well. Japanese orthography employs Chinese characters alongside two visually distinct syllabaries called Hiragana and Katakana, which are phonologically equivalent. Al- though Chinese characters are often used for the representation of words or morphemes, there are also many words written usually in one of the syllabic Kana scripts: Foreign loanwords are usually represented with Katakana, whereas another class of words, for example, botanical names or words denoting native Japanese food items, are usually written with Hiragana. Words that are regularly or exclusively written with Kana characters form a subset of the vocabulary. In general, they are written, and judged to be visually very familiar, in either one of the two syllabaries. Rewritten in the other syllabary they are judged as being visually very unfamiliar (for an overview of stimulus properties, see Table 1). For example, the Japanese loanword for video, pronounced bi.de.o, is usually written with Katakana: ビデオ. The same word is rated as being visually very unfamiliar by skilled readers of Japanese when it is rewritten with Hiragana: オ゙でお (for examples of the stimuli, see Figure 1). Both syllabaries are used approximately equally frequently and GPC rules are identical for both scripts.

If visually familiar Kana words are read using the direct visual route, differences between visually familiar and unfamiliar words observed in a brain imaging experiment might therefore indicate the neural correlates of the direct visual pathway. However, because Japanese Kana orthography is perfectly regular, it has been hypothesized in the past that native speakers of Japanese might make no use at all of the direct visual pathway and rely exclusively on the phonological assembly route (e.g., Yamadori, 2000; Iwata, 1984). In a task of reading Kana words aloud they might even skip lexical access entirely. This is known as the strictest version of the orthographic depth hypothesis (Katz \& Frost, 1992). If this hypothesis in this strict sense were true, an experiment using Japanese Kana words would not succeed in yielding information about the visual pathway. Behavioral studies, however, do not support this version of the orthographic depth hypothesis. Visual familiarity and the lexical status of Kana words have been found to facilitate reaction

Table 1. Mean Values of the Kana Word Properties for their Presentation in Familiar and Unfamiliar Script

\begin{tabular}{lcc}
\hline Script & Word Familiarity & Script Familiarity \\
\hline Katakana & & \\
Familiar & 6.5 & 5.0 \\
Unfamiliar & 6.4 & 1.3 \\
Hiragana & & \\
Familiar & 6.3 & 4.7 \\
Unfamiliar & 6.5 & 1.2 \\
\hline
\end{tabular}

Note. Word familiarity: subjective rating (7-point scale) of spoken and written word familiarity. Script familiarity: subjective rating (5-point scale) of the familiarity of the word written in one of the Kana scripts, as obtained from the NTT database (Amano \& Kondo, 2000) 
Figure 1. An example of the Kana stimuli.

\begin{tabular}{|c|c|c|c|}
\hline Hiragana familiar & 3 & maguro & tuna \\
\hline Hiragana pseudoword & $d t^{*} b$ & abari & \\
\hline Hiragana unfamiliar & 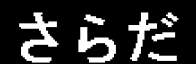 & sarada & salad \\
\hline Katakana familiar & & sarada & salad \\
\hline Katakana pseudoword & 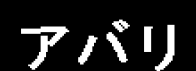 & abari & \\
\hline Katakana unfamiliar & ハナヒ & hanabi & fireworks \\
\hline
\end{tabular}

times in several tasks, including reading aloud (e.g., Besner \& Hildebrand, 1987; Besner \& Smith, 1992) and phonological lexical decision (e.g., Usui, 1998; Kawakami, 1993; Yamada, Imai, \& Ikebe, 1990; Hatta, Katoh, \& Kirsner, 1984; see also Table 2). The effects of word-level visual familiarity might therefore be interpreted as evidence for a visual word form lexicon for highly familiar Kana words. Given the complexity and variability of the Japanese script it is possible, however, that only a small subset of the vocabulary, namely, extremely frequent Kana words are stored in the visual word form lexicon. The dual-route model might therefore have only a limited range of applicability and thus is not representative for Kana reading in general. It should be noted that the evidence cited here and the results of the present study do not support or refute the more commonly used

Table 2. Response Times (in msec) and Error Rates as Obtained in the Two Behavioral Experiments

\begin{tabular}{clll}
\hline \multirow{4}{*}{ Task } & \multicolumn{3}{c}{ Word Type } \\
\cline { 2 - 4 } Naming & Familiar & Unfamiliar & Pseudoword \\
Hiragana & $523(25.6)$ & $548(26.2)$ & $665(50.1)$ \\
& $1.85 \%$ & $2.78 \%$ & $5.32 \%$ \\
Katakana & $517(18.2)$ & $622(43.6)$ & $669(46.5)$ \\
& $1.62 \%$ & $4.17 \%$ & $4.63 \%$ \\
pLDT & & & $760(70.3)$ \\
Hiragana & $510(30.0)$ & $569(42.7)$ & $3.49 \%$ \\
& $0.47 \%$ & $2.09 \%$ & $780(71.7)$ \\
Katakana & $492(27.1)$ & $715(73.0)$ & $3.95 \%$ \\
\hline & $0.47 \%$ & $12.33 \%$ &
\end{tabular}

Note. Standard errors are given in parentheses, error percentages below the response times.

${ }^{a}$ pLDT $=$ phonological lexical decision task. weaker version of the orthographic depth hypothesis, namely, that readers of regular orthographies rely more strongly on the assembly route than readers of irregular orthographies. There is indeed some evidence for behavioral (see, for a review, Katz \& Frost, 1992) as well as neural (Paulesu et al., 2000) differences in the processing of regular and irregular orthographies.

The present study is designed to address the following questions: first, whether there are stable differences in brain activation patterns between visually familiar words, visually unfamiliar words, and pseudowords; second, given that differences are found, if they can interpreted in the framework of the dual-route model; additionally, whether areas can be identified that respond selectively to words and pseudowords. Lastly, we are interested in how much the results depend on the task (silent articulation, phonological lexical decision).

The first fMRI experiment reported in the present study employed visually familiar and unfamiliar words, as well as pseudowords in a silent articulation task using an event-related design. If the dual-route model is valid, pseudowords as well as unfamiliarly written words will have to be phonologically assembled, whereas the pronunciation of visually highly familiar words can be accessed directly. Stronger activations for visually unfamiliar words and pseudowords might therefore indicate regions involved in the processing of grapheme to phoneme conversion.

It is unclear, however, what regions might be associated with the direct visual route. If access to the visual word form lexicon is conceptualized as a serial search process (e.g., Forster, 1976), visually unfamiliar words and pseudowords should yield stronger activations than visually familiar words. In an event-related design with randomized materials as in the present study, it can be assumed that access to the visual word form lexicon is always attempted. In the case of visually highly familiar words, the search can terminate earlier. On the other hand, it could be predicted that a specialized population of neurons displays increased activation when a visually familiar word stimulus is encountered (see, Polk \& Farah, 2002, for a similar argument). In this case, visually 
highly familiar words should activate a word form recognition area more strongly than visually unfamiliar words or pseudowords, similar to, for example, the fusiform face recognition area (Kanwisher, McDermot, \& Chun, 1997).

The second fMRI experiment, conducted using the same stimuli but a different task (phonological lexical decision), provided an estimate of the extent to which brain activations observed in Experiment 1 can be attributed to the task rather than to differences in the stimulus materials. In this task, visually familiar words can be judged correctly by looking them up directly in the visual word form lexicon. In the case of visually unfamiliar words and pseudowords, participants have to assemble the pronunciation of the stimulus and check it against the auditory word form lexicon to give a correct answer. As elaborated in the task analysis in the Discussion section, both tasks require very similar processing steps. Additionally, two off-line behavioral experiments were conducted to test the stimulus materials in a task of reading aloud and phonological lexical decision

\section{RESULTS}

\section{Behavioral Experiments}

In the first experiment participants were instructed to read aloud, as fast and correctly as possible, familiarly and unfamiliarly written words and pseudowords presented on a monitor. The factors script (Katakana, Hiragana) and word type (familiar, unfamiliar, and pseudoword) yielded six conditions in total. Only naming latencies for correct answers between 250 and $2000 \mathrm{msec}$ (2504 of 2592 data points) were analyzed (see Table 2) and entered into a repeated measures ANOVA with the two factors script and word type. There was a significant main effect of the factor word type, $F(1,11)=15.19$, $S E M=692, p<.01$, due to the slower reaction times to visually unfamiliar words and pseudowords as compared to visually familiar words. The significant main effect of the factor script, $F(2,22)=80.14, S E M=1667, p<.001$, and interaction of both factors, $F(2,22)=12.67$, SEM $=$ $888, p<.001$, are due to the significant difference between visually unfamiliar Katakana and Hiragana words, $t(11)=6.33, p<.001$. Reaction times to visually familiar words or pseudowords did not differ significantly between Hiragana and Katakana. The longer reaction times to visually unfamiliar Katakana are most likely caused by our choice of stimuli. Because rewriting familiar Hiragana words with Katakana would have yielded stimuli that have medium instead of very low visual familiarity ratings, we used words usually written with Chinese characters for the Katakana unfamiliar condition (see Besner \& Hildebrandt, 1987; Hatta et al., 1984, for a similar choice of stimuli). Words usually written with Chinese characters have been found to be rated visually highly unfamiliar when presented in Katakana (Amano \&
Kondo, 2000). However, this condition proved to be more difficult for the Japanese participants even though overall word familiarity was matched to the Katakana familiar condition. The overall familiarity effect is significant for Katakana, $t(11)=7.39, p<.001$, as well as for Hiragana, $t(11)=3.94, p<.01$, when analyzed separately.

In the second experiment, the same materials and procedure were used with a phonological lexical decision task. Only reaction times for correct answers between 250 and $2000 \mathrm{msec}$ (2494 of 2592 data points) were analyzed (see Table 2 ) and entered into a repeated measures ANOVA with the two factors script and word type. Again there was a significant main effect of the factor word type, $F(1,11)=35.72$, SEM $=1238, p<.001$, due to the slower reaction times to visually unfamiliar words and pseudowords. The significant main effect of the factor script, $F(2,22)=63.40, S E M=6740, p<.001$, and the significant interaction of both factors, $F(2,22)=$ 24.50, SEM $=1765, p<.001$, are, as in the naming experiment, due to the longer reaction times for visually unfamiliar Katakana words, $t(11)=6.29, p<.001$. Again, visually familiar words were judged significantly faster than visually unfamiliar words in both scripts, Katakana: $t(11)=7.59, p<.001$; Hiragana: $t(11)=5.97, p<.001$. The observed pattern of response times is very similar to the results of the first experiment.

\section{fMRI Experiments}

\section{Experiment 1: Silent Articulation Task}

Ten participants took part in this experiment performing a silent articulation task. In an event-related design, the same materials were used as in the behavioral experiments. The six conditions of the experiment were collapsed over the factor script yielding three conditions, which will be referred to in short as "familiar," "unfamiliar," and "pseudoword." Six contrasts were calculated in total.

Only visually familiar words can be recognized in the visual word form lexicon, while visually unfamiliar words and pseudowords have to be assembled. It can then be expected that visually unfamiliar words and pseudowords will draw more strongly on processing resources for phonological assembly and encoding. We will therefore first report the results of the comparisons unfamiliar $>$ familiar and pseudoword $>$ familiar. These two comparisons yielded similar results (top half of Figure 2, Table 3). In both comparisons, a brain area in the left inferior frontal gyrus, corresponding to $\mathrm{BA} 9 / 44^{1}$ and extending ventrally to BA 47 was found to be significantly activated, as well as a corresponding area in the right hemisphere and a region in the left inferior parietal lobule. An additional activation was found for the comparison pseudoword $>$ familiar in the medial frontal lobe corresponding to BA 6/8, SMA. An area in the right cuneus (BA 19) was found to be activated in the comparison unfamiliar $>$ familiar. 
Figure 2. Renderings given for the comparisons unfamiliar $>$ familiar and pseudoword $>$ familiar, revealing activations related to phonological encoding, for the silent articulation task (Experiment 1) and the phonological lexical decision task (Experiment 2). Renderings are thresholded at $p<.001$, uncorrected, at voxel level (left is left).

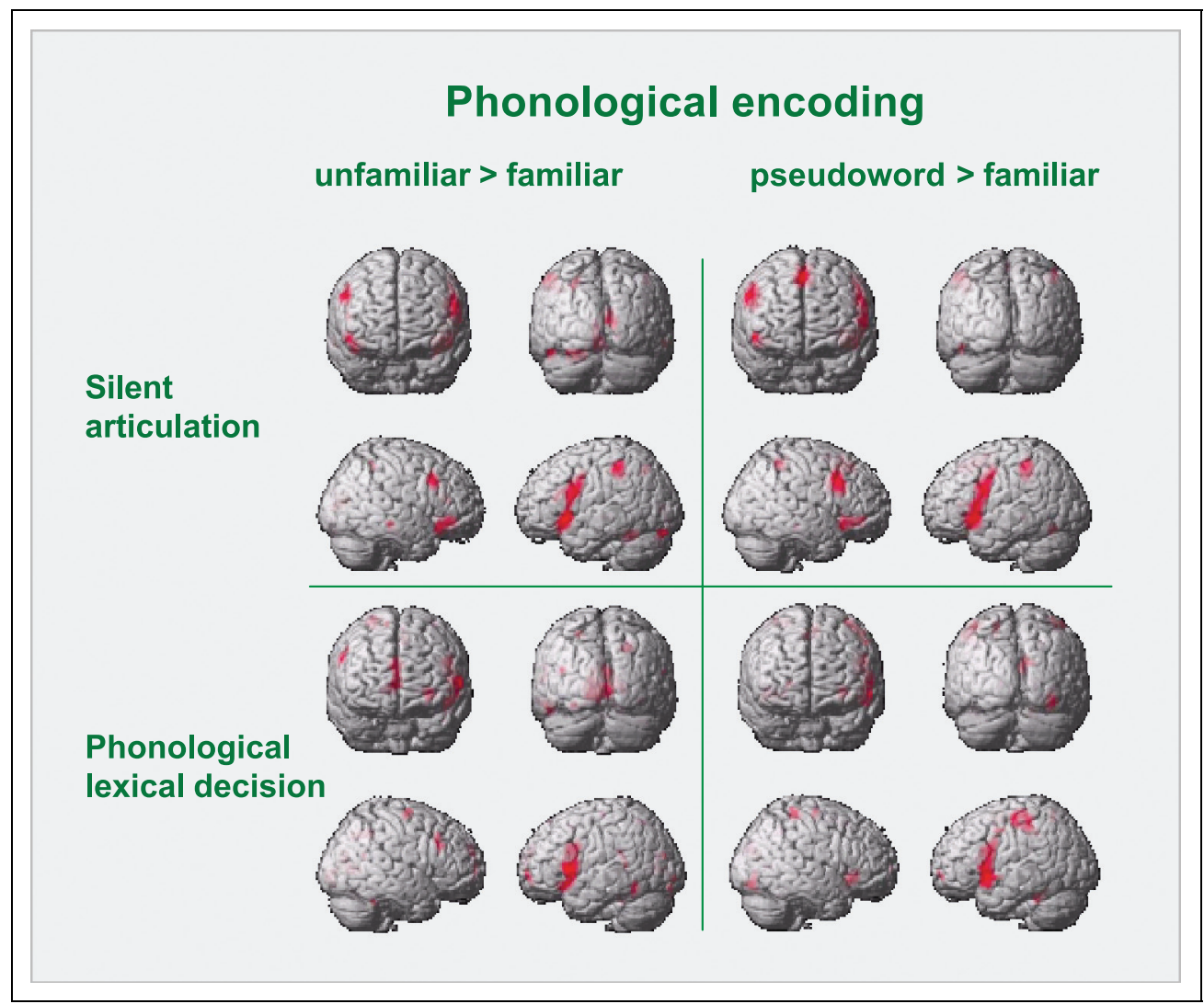

Because familiarly as well as unfamiliarly written words allow access to word-level lexical properties such as meaning, brain areas related to the processing of lexical information or meaning might be found to be more active in the case of words as compared to pseudowords. We will therefore report in the following
Figure 3. Renderings given for the comparisons familiar > pseudoword and unfamiliar $>$ pseudoword, revealing activations related to lexico-semantic processing, for the silent articulation task (Experiment 1) and the phonological lexical decision task (Experiment 2). Renderings are thresholded at $p<.001$, uncorrected, at voxel level (left is left).

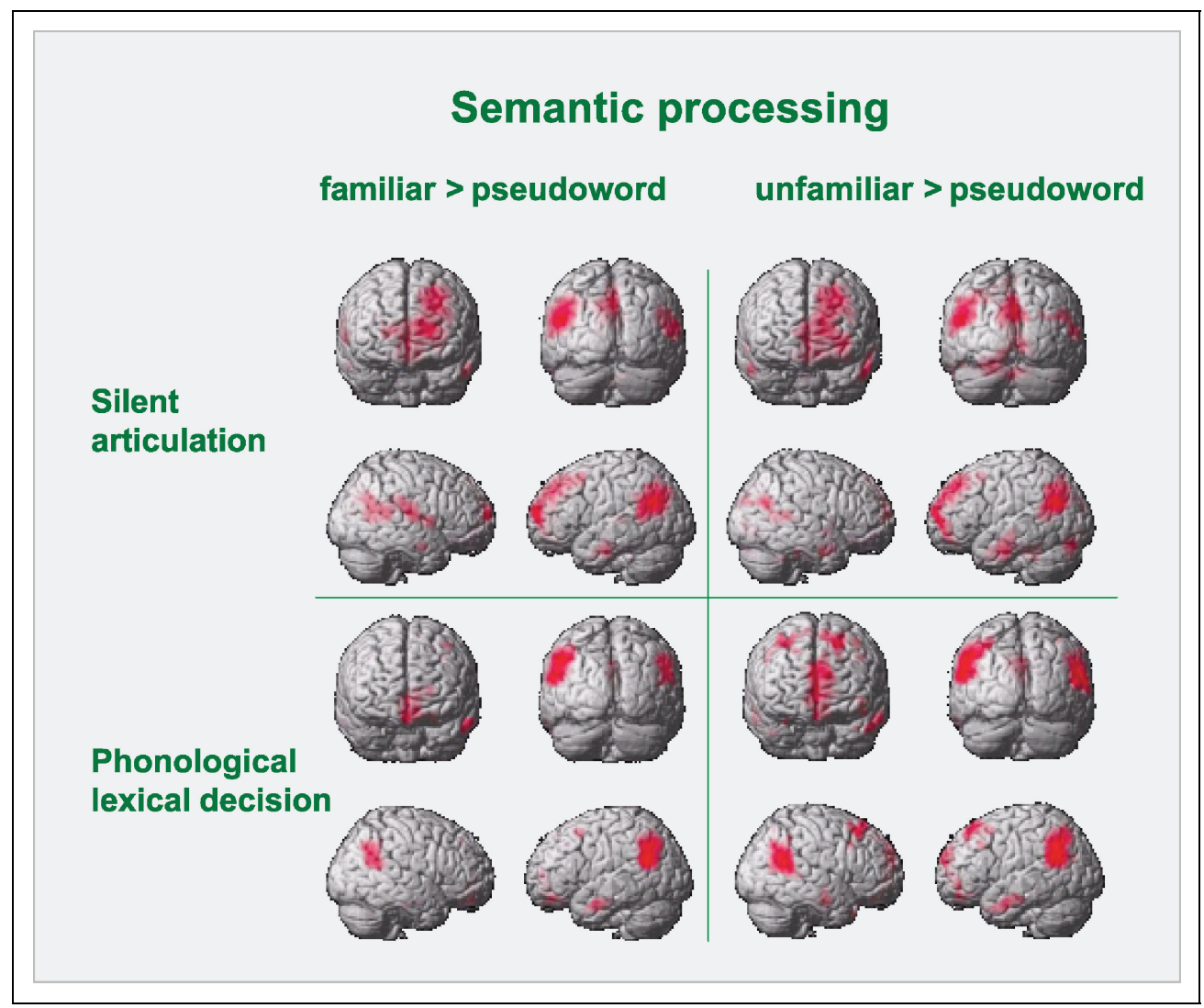


the results of the two comparisons familiar $>$ pseudoword and unfamiliar $>$ pseudoword. Both comparisons yielded a similar pattern of results (top half of Figure 3, Table 3). An extended left temporo-parietal area (BA 39/ 40) was found to be more strongly activated for visually familiar as well as visually unfamiliar words than in the case of pseudowords, as well as a homologous area in the right hemisphere, a region in the left inferior temporal gyrus (BA 20/21), a medial area in the parietal lobe, with a focus in the posterior cingulate gyrus (BA 31), and an area in the left middle frontal gyrus (BA 9/10). Additional activations found to be significant only in the comparison unfamiliar $>$ pseudoword were located in the right inferior temporal gyrus, right and left occip-

Table 3. Peak Activations for Clusters Found to Be Significantly Activated in the Silent Articulation Task (Experiment 1)

\begin{tabular}{|c|c|c|c|c|c|c|c|c|c|c|}
\hline Brain Area & $x$ & $y$ & $z$ & $Z_{\max }$ & $k^{\mathrm{a}}$ & $x$ & $y$ & $z$ & $Z_{\max }$ & $k^{\mathrm{a}}$ \\
\hline & \multicolumn{5}{|c|}{ Unfamiliar $>$ Familiar } & \multicolumn{5}{|c|}{ Pseudoword $>$ Familiar } \\
\hline \multicolumn{11}{|l|}{ Frontal } \\
\hline 1. IFG (BA 44/45) & -50 & 10 & 23 & 5.71 & 305 & -56 & 10 & 27 & Inf. & 385 \\
\hline r. IFG (BA 47) & 45 & 32 & -11 & 4.57 & 96 & 48 & 40 & -7 & 4.45 & 121 \\
\hline r. MFG (BA 9) & 50 & 19 & 31 & 4.44 & 51 & 56 & 19 & 31 & 5.39 & 157 \\
\hline SMA (BA 6/8) & & & & & & 6 & 26 & 45 & 5.84 & 230 \\
\hline
\end{tabular}

Temporo-parietal

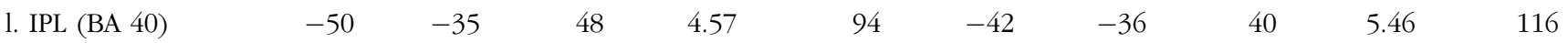

Occipital

r. Cun. (BA 18)

$\begin{array}{llll}12 & -81 & 12 & 4.23\end{array}$

71

Familiar $>$ Pseudoword

Unfamiliar $>$ Pseudoword

Frontal

$\begin{array}{llllll}\text { l. MFG (BA 10) } & -24 & 61 & 6 & 7.33 & 1183\end{array}$

1. MedFG (BA 10)

$-15$

$\begin{array}{lll}58 & 2 & 5.49\end{array}$

997

Temporal

1. MTG (BA 20/21)

$\begin{array}{llll}-53 & -13 & -20 & 6.12\end{array}$

86

$\begin{array}{rrrrr}-59 & -13 & -20 & 6.22 & 152 \\ 59 & -1 & -23 & 4.35 & 48 \\ -39 & -30 & -20 & 4.24 & 84 \\ 42 & -33 & -15 & 3.99 & 55\end{array}$

r. FusiG (BA 20)

Temporo-parietal

1. IPL (BA 39/40)

$$
-50 \quad-63
$$

24

Inf.

1470

$-50$

$-63$

24

Inf.

1286

r. IPL (BA 39/40)

$65-17$

$\begin{array}{ll}13 & 5.97\end{array}$

654

48

$-63$

24

4.58

158

Occipital

Post. C. (BA 31)

$\begin{array}{ll}-6 & -57\end{array}$

20

6.41

$1470^{\mathrm{b}}$

$-6$

$-57$

24

6.29

$1286^{\mathrm{b}}$

1. FusiG (BA 19)

$-21$

$-82$

$-17$

5.12

Note. The statistical parameter map is thresholded at $p<.001$, uncorrected at voxel level. Only clusters significant at a threshold of $p<.05$, corrected at cluster level, are reported. Coordinates and Brodmann's areas conform to the atlas of Talairach and Tournoux (1988). IFG $=$ inferior frontal gyrus, $\mathrm{MFG}=$ middle frontal gyrus, $\mathrm{MedFG}=$ medial frontal gyrus, $\mathrm{SMA}=$ supplementary motor area, $\mathrm{MTG}=$ middle temporal gyrus, $\mathrm{IPL}=$ inferior parietal lobule, Post. $\mathrm{C} .=$ posterior cingulate, $\mathrm{Cun} .=$ cuneus, $\mathrm{FusiG}=$ fusiform gyrus.

${ }^{\mathrm{a}}$ Cluster size.

${ }^{\mathrm{b}}$ The activation of the posterior cingulate (BA 31) is part of a bigger cluster. 
ital fusiform gyrus (BA 18/19) and in the right and left temporal fusiform gyrus (BA 36/37). The remaining two comparisons familiar $>$ unfamiliar and pseudoword $>$ unfamiliar did not yield any suprathreshold clusters.

Given the significant difference between the two scripts regarding the unfamiliar condition, it is possible that the pattern of results does not generalize over both scripts. To investigate the symmetry of the results for both scripts, selective averages of the time course of the BOLD response for all six experimental conditions are given in Figure 4.

\section{Experiment 2: Phonological Lexical Decision Task}

The same experimental procedure and materials were used as in Experiment 1, using a phonological lexical decision task. The six conditions were again collapsed over the factor script yielding three conditions: familiar, unfamiliar, and pseudoword. Six linear contrasts were calculated.

As in Experiment 1, first the results of the comparisons unfamiliar $>$ familiar and pseudoword $>$ familiar are reported. These two comparisons yielded a similar pattern of results (bottom half of Figure 2, Table 4), similar to the results of Experiment 1 . In both comparisons, areas in the left inferior frontal gyrus, corresponding to BA $44 / 45$ and BA 47 , were found to be more strongly activated for unfamiliarly written words nd pseudowords than in the case of familiarly written words. Additional activations were found for the comparison unfamiliar $>$ familiar in the anterior medial frontal gyrus, the right superior parietal lobule, and a medial occipital area. In the comparison pseudoword $>$ familiar additional activations were found in the SMA (BA 6), and in the left inferior parietal lobule.

As in Experiment 1, the results of the comparisons familiar $>$ pseudoword and unfamiliar $>$ pseudoword might indicate brain areas involved in lexico-semantic processing. These comparisons yielded results (bottom half of Figure 3, Table 4) that also resemble the results of Experiment 1. In both comparisons, an extended left temporo-parietal area (BA 39/40) was found to be strongly activated, as well as a homologous region in the right hemisphere. Also, a region in the left inferior temporal gyrus (BA 20/21) was found to be significantly activated in both comparisons, as well as areas in the medial frontal lobe (BA 10/11), and a medial area in the occipital lobe with a focus in the posterior cingulate (BA 31). The remaining two comparisons familiar > unfamiliar and pseudoword $>$ familiar yielded no suprathreshold clusters.

\section{Joint Analysis of Experiment 1 and Experiment 2}

To investigate possible activation differences between the two tasks, a random effects analysis was calculated.
However, activation differences between the tasks did not surpass the significance threshold for any of the six comparisons.

\section{DISCUSSION}

\section{An Overview over the Principal Results}

The first goal of the present study was to investigate whether the behavioral differences between visually highly familiar and unfamiliar Kana words and pseudowords give rise to a stable pattern of activation differences in the brain. The very systematic differences between visually familiar and unfamiliar words found consistently over two different tasks support the assumption that skilled readers of Japanese process these stimulus types differently, possibly in a way as assumed in the dual-route model of reading.

Our second focus of interest was to identify brain regions that might correspond to the two pathways assumed in the dual-route model. According to the dual-route model, the pronunciation of visually unfamiliar words and pseudowords has to be constructed sequentially, whereas the pronunciation of visually highly familiar words can be accessed directly. A coherent region in the left inferior frontal gyrus corresponding to BA 44, 45, and 47 was found to be more strongly activated for unfamiliarly written words and pseudowords than for visually highly familiar words. A corresponding activated area was observed in the right inferior frontal gyrus. These areas have been associated, among others, with sequential phonological encoding (e.g., Fiez et al., 1999). It is possible that the activation differences observed here reflect a gradual rather than a categorical difference with regard to the use of the phonological assembly route. Although the assembly route is used less for visually highly familiar words, it is drawn upon more strongly in the case of visually unfamiliar words and pseudowords. The activation found in this area might thus also reflect the greater difficulty to process these words, as indicated by the longer reaction times.

It seems to be more difficult, however, to identify correlates of the direct visual pathway in the present study. If a word recognition area existed in the brain similar to other object recognition areas identified so far, more activation is expected in this area for visually familiar than for unfamiliar or unknown word stimuli. However, no active region was identified in the comparison familiar > unfamiliar. Alternatively, it is conceivable that visually familiar words allow the early termination of a search process and should therefore lead to less activation in the visual word form area than visually unfamiliar words and pseudowords. All areas more active for visually unfamiliar and pseudowords are then also candidates for a visual word form area.

Furthermore, we were interested in whether there would be a difference in brain activations reflecting the 
lexical status of the stimuli (i.e., the stimulus being a word or a pseudoword). Compared to pseudowords, words gave rise to a higher level of activation in the left and in the right temporo-parietal region, in the middle part of the left middle/inferior temporal gyrus (BA 20/
21), and in the posterior cingulate (BA 31) in all four comparisons. These activations were unaffected by visual familiarity. It is therefore possible that these regions represent semantic or lexical processes, for example, an auditory word form lexicon. The observed direction of
Figure 4. Selected averages of the time courses of the BOLD response for all six conditions. The heading "phonological encoding" refers to the two comparisons unfamiliar > familiar and pseudoword $>$ familiar. The heading "lexico-semantic processing" refers to the two comparisons familiar $>$ pseudoword and unfamiliar $>$ pseudoword. The ROI plots are given as the average of all voxels surpassing the significance threshold in both comparisons under each heading and for each task.

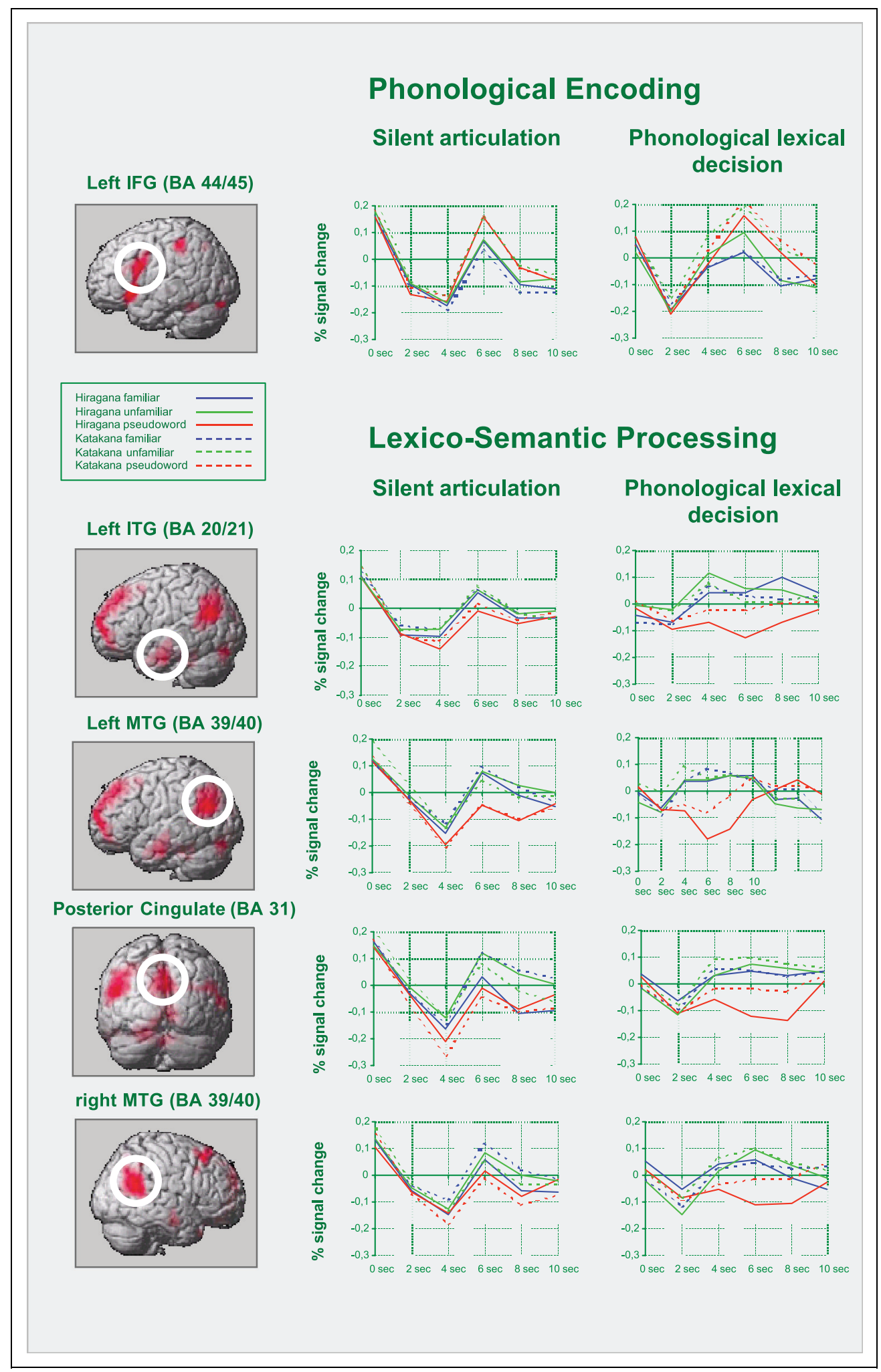


Table 4. Peak Activations for Clusters Found to Be Significantly Activated in the Phonological Lexical Decision Task (Experiment 2)

\begin{tabular}{|c|c|c|c|c|c|c|c|c|c|c|}
\hline Brain Area & $x$ & $y$ & $z$ & $Z_{\max }$ & $k^{\mathrm{a}}$ & $x$ & $y$ & $z$ & $Z_{\max }$ & $k^{\mathrm{a}}$ \\
\hline & \multicolumn{5}{|c|}{ Unfamiliar $>$ Familiar } & \multicolumn{5}{|c|}{ Pseudoword $>$ Familiar } \\
\hline \multicolumn{11}{|l|}{ Frontal } \\
\hline 1. IFG (BA 44/45) & -53 & 15 & 4 & 5.00 & 198 & -53 & 11 & -10 & 5.39 & 247 \\
\hline r. IFG (BA 47) & & & & & & 39 & 26 & -6 & 4.32 & 61 \\
\hline MedFG (BA 10) & 6 & 61 & 2 & 4.04 & 59 & & & & & \\
\hline SMA (BA 6) & & & & & & -3 & -7 & 42 & 4.20 & 75 \\
\hline \multicolumn{11}{|l|}{ Temporo-parietal } \\
\hline r. SPL (BA 7) & 21 & -48 & 36 & 4.51 & 57 & & & & & \\
\hline 1. IPL (BA 40) & & & & & & -45 & -30 & 44 & 4.43 & 141 \\
\hline \multicolumn{11}{|l|}{ Occipital } \\
\hline \multirow[t]{2}{*}{ r. Cun. (BA 18) } & 15 & -67 & 8 & 4.35 & 225 & & & & & \\
\hline & \multicolumn{5}{|c|}{ Familiar > Pseudoword } & \multicolumn{5}{|c|}{ Unfamiliar $>$ Pseudoword } \\
\hline \multicolumn{11}{|l|}{ Frontal } \\
\hline 1. MedFG (BA 10/11) & -3 & 48 & -21 & 6.49 & 324 & -3 & 53 & 2 & 5.88 & 477 \\
\hline 1. SFG (BA 8) & & & & & & -21 & 37 & 44 & 4.59 & 278 \\
\hline \multicolumn{11}{|l|}{ Temporal } \\
\hline 1. MTG (BA 21) & -56 & -1 & -23 & 5.55 & 55 & -53 & 7 & -28 & 5.14 & 83 \\
\hline \multicolumn{11}{|l|}{ Temporo-parietal } \\
\hline 1. IPL (BA 39/40) & -50 & -54 & 24 & 7.58 & 344 & -50 & -57 & 32 & 7.06 & 427 \\
\hline r. IPL (BA 39/40) & 53 & -57 & 28 & 4.93 & 202 & 56 & -54 & 20 & 6.44 & 295 \\
\hline \multicolumn{11}{|l|}{ Occipital } \\
\hline Post. C. (BA 31) & 3 & -51 & 28 & 4.85 & 175 & 0 & -51 & 19 & 6.65 & 345 \\
\hline
\end{tabular}

Note. The statistical parameter map is thresholded at $p<.001$, uncorrected at voxel level. Only clusters significant at a threshold of $p<.05$, corrected at cluster level, are reported. Coordinates and Brodmann's areas conform to the atlas of Talairach and Tournoux (1988). IFG $=$ inferior frontal gyrus, $\mathrm{MFG}=$ middle frontal gyrus, $\mathrm{MedFG}=$ medial frontal gyrus, $\mathrm{SMA}=$ supplementary motor area, $\mathrm{MTG}=$ middle temporal gyrus, $\mathrm{IPL}=$ inferior parietal lodule, $\mathrm{SPL}=$ superior parietal lobule; Post. $\mathrm{C} .=$ posterior cingulate, Cun. $=$ Cuneus; FusiG $=$ fusiform gyrus.

${ }^{\mathrm{a} C l u s t e r}$ size.

the activation (words activate more than nonwords), however, does not match the idea of a resource-intensive lexical search, but the behavior of an object recognition area. In the case of lexical search, higher activations are expected for pseudowords than for words. It is also possible that these areas represent word meaning rather than lexical access, as proposed by Binder et al. (2003). The present study, however, cannot rule out either possibility.

Third, we were interested in whether the observed activation differences between the conditions generalize over tasks. The absence of significant differences be- tween the two tasks for any of the six comparisons indicates that the stimuli were similarly processed in both tasks and that differences between the conditions can be attributed to differences in the stimulus materials. In the following, a more detailed task analysis is provided to illustrate the similarities between the two tasks.

\section{Task Analysis}

In a task of reading aloud or silent articulation, first the visual features of the stimuli are analyzed. After the identification of individual characters, phoneme to 
grapheme conversion can be performed. In a perfectly regular orthography, the utterance can be initiated directly after grapheme to phoneme conversion. The behavioral data, however, suggest that skilled readers of Japanese do not rely exclusively on the assembly route in a task of reading aloud. They attempt to access the word in the mental lexicon before utterance. Finding a match in the visual word form lexicon then translates into faster word recognition and naming times for familiarly written words. The search in this lexicon will time-out (Taylor \& Lupker, 2001), however, for unfamiliarly written words and pseudowords. Their pronunciation has to be assembled using GPC rules. Similarly, the faster reading times for visually unfamiliar words compared to pseudowords indicate that the reader also makes an attempt to look up the assembled candidate in the auditory word form lexicon. In the case of pseudowords, the search in the auditory word form lexicon will finally time-out, resulting in longer naming latencies for pseudowords. However, given that Japanese Kana orthography is perfectly regular, why should the reader retrieve a word in the visual and the auditory word form lexicon in a silent articulation task? A reason for this strategy might lie in the underspecification of prosodic parameters by Japanese Kana orthography, such as correct tone accent. Tone accent is often irregular and a lexical property of the Japanese vocabulary (cf., Vance, 1987). An assembled articulatory approximation of the word might therefore not satisfy a skilled reader intending to read correctly. An additional gain of this strategy might be the reduction in articulatory processing load when a word is recognized. Then a whole-word motor program could be used for its articulation, instead of the effortful sequential assembly of smaller articulatory elements in the case of pseudowords. The randomization of the stimuli and the use of both scripts in all conditions prevented the participants from skipping the search in the visual and auditory word form lexicon.

In the phonological lexical decision task, after processing the visual features of the stimuli, grapheme to phoneme conversion can be performed. Familiarly written words can be directly recognized in the visual word form lexicon. In contrast to the silent articulation task, the retrieval of the correct pronunciation is not necessary since only a categorization response is required. Similar to the silent articulation task, pseudowords and unfamiliarly written words have to be phonologically assembled and checked against the auditory word form lexicon. A pseudoword response can be given only after the search in the auditory word form lexicon has ended unsuccessfully. This task analysis, therefore, matches the ordering of phonological lexical decision times found in the present study and elsewhere. The two tasks used in the present study thus involve very similar processing steps. However, there are also differences. Accessing the pronunciation of visually familiar words is only obli- gatory in the silent articulation task, although it might be accessed automatically in the phonological lexical decision task as well. The silent articulation task requires an articulatory response, whereas a decision and a manual categorization response is required in the phonological decision task.

\section{Visual Familiarity and Phonological Processing}

Areas in the left inferior frontal gyrus (BA 44/45), including the anterior insula, have been repeatedly associated with phonological processing in neuroimaging studies (Fiez et al., 1999; Herbster, Mintun, Nebes, \& Becker, 1997). Compared to words, these areas have been found to be activated more strongly by pseudowords (Hagoort et al., 1999; Herbster et al., 1997; Rumsey et al., 1997), words with a low frequency of occurrence (Fiebach, Friederici, Müller, \& von Cramon, 2002; Fiez et al., 1999), words written with mirrored letters (Dong et al., 2000), and, as observed in the present study, words written in an unfamiliar script. This is compatible with the view that a common mechanism mediates the pronunciation of both pseudowords, words with a low frequency of occurrence and unfamiliarly written words, namely, effortful sequential phonological assembly by grapheme to phoneme conversion. Another area that has been associated with GPC processing is the posterior part of the superior temporal gyrus at the temporo-parietal junction (Paulesu et al., 2000; Price, 2000). In the present study, however, this area was not found to be more active in the case of visually unfamiliar words and pseudowords as compared to visually familiar words.

\section{Possible Locations of the Visual Word Form Lexicon}

A number of very diverse brain areas have been proposed as possible locations of a visual word form lexicon. One of these candidate areas is the posterior part of the middle and superior temporal gyrus, including the angular gyrus (Paulesu et al., 2000; Beauregard et al., 1997; Menard, Kosslyn, Thompson, Alpert, \& Rauch, 1996; Howard et al., 1992). Recently, however, this region has been associated with semantic rather than orthographic processing (see, for a review, Binder, 1999; Price, Indefrey, \& van Turennout, 1999). This view is corroborated by the present study, which reports a stronger activation of this region for words than for pseudowords independent of orthographic familiarity.

Other studies suggest that the visual word form lexicon is located in the posterior part of the left inferior temporal gyrus, including the fusiform gyrus (e.g., Cohen et al., 2000; Paulesu et al. 2000; Price, 2000; Law et al., 1991). Neuropsychological evidence from patients with pure alexia suggests that this region indeed plays a crucial role in reading (Damasio \& Damasio, 1983). In this region, brain areas have been identified that respond selectively to highly specific complex visual stim- 
uli such as faces (Kanwisher et al., 1997) or places (Epstein \& Kanwisher, 1998), and it is regarded functionally as a part of the ventral processing stream of object recognition (Mishkin, Ungerleider, \& Macko, 1983). Object recognition areas usually exhibit stronger activation when a matching stimulus is presented. Therefore, a visual word form area located in this region is expected to also display more activation when a visually familiar word is encountered, than in the case of a pseudoword or visually unfamiliar word (Polk \& Farah, 2002). Functional imaging evidence, however, is divergent even in this respect. Some studies report stronger activation for words than for pseudowords (Fiebach et al., 2002; Herbster et al., 1997), whereas others report less activation (Paulesu et al., 2000; Fiez et al., 1999; Hagoort et al., 1999). In the present study, neither a difference between words and pseudowords nor an influence of orthographic familiarity was observed with regard to this region. This is compatible with other studies, which also report no difference between words of low and high frequency of occurrence (Fiebach et al., 2002; Fiez et al., 1999), and between case alternated words and normally written words (Polk \& Farah, 2002; Mayall, Humphreys, Mechelli, Olson, \& Price, 2001; Xu et al., 2001) for any area in this region. It is possible, however, that this region has a highly specialized and intricate functional architecture difficult to investigate given the limited spatial resolution and signal-to-noise ratio of present imaging methods. Areas in this region have been identified which are specialized in the processing of letters or digits (Polk et al., 2002), an abstract or case-independent representation of written words (Polk \& Farah, 2002; Dehaene et al., 2001), and orthographic regularity (Cohen et al., 2000).

A third region put forward as a possible location of the visual word form lexicon is the extrastriate area in the occipital lobe (e.g., Pugh et al., 1996; Petersen et al., 1988; Petersen, Fox, Snyder, \& Raichle, 1990). Results are again diverse: Some studies report a stronger activation for words than for pseudowords (Fiebach et al., 2002; Petersen et al., 1988, 1990), others report less activation (Hagoort et al., 1999) or failed to find any difference (e.g., Xu et al., 2001; Herbster et al., 1997; Howard et al., 1992). It is possible that this area is specialized with regard to more basic aspects of letter processing: Activation seems to depend on string length rather than being letter specific (Indefrey et al., 1997). In the present study, unfamiliarly written words but not pseudowords were found to activate this area more strongly than familiarly written words. However, in the light of the above-mentioned evidence it might not be justified to conclude that this area is specific for the recognition of whole word forms. It is possible that the observed activation in this area is due to basic visual processes modulated by attention. In the case of randomized trial presentation, the visual analysis of an unfamiliar word might be reemphasized if a word is recognized in the auditory word form lexicon but not in the visual word form lexicon.

\section{Lexicality and Semantic Processing}

Several imaging studies have compared the processing of words to pseudowords in tasks such as reading aloud (e.g., Fiez et al., 1999; Hagoort et al., 1999; Herbster et al., 1997; Rumsey et al., 1997; Petersen et al., 1988), lexical decision (Binder et al., 2003; Fiebach et al., 2002), or rhyming (Xu et al., 2001). Although often an activation of the left inferior frontal gyrus is reported for pseudowords compared to words, results are less consistent for the comparison of words to pseudowords. Some studies do not report any region significantly more activated by words than by pseudowords (Xu et al., 2001; Tagamets, Novick, Chalmers, \& Friedman, 2000; Fiez et al., 1999; Rumsey et al., 1997). In the present study, a set of areas consisting of a left and right temporo-parietal area (BA 39/40), a small region in the middle part of the left middle/inferior temporal gyrus (BA 21/20), and the posterior cingulate (BA 31) was observed to be more active for words than for pseudowords, independent of visual familiarity. Some of these areas replicate the results of other studies using tasks of reading or lexical decision: An activation in the temporo-parietal region is reported by Hagoort et al. (1999), and the middle part of the left middle/inferior temporal gyrus by Fiebach et al. (2002). The whole set of regions reported here, including the activation in the posterior cingulate and frontal areas, has been observed in difficult semantic judgment tasks (Binder et al., 1997, 1999; Démonet et al., 1992). In the recent study of Binder et al. (2003), the same set of regions has been identified for a lexical decision task, using orthographically controlled words and pseudowords in an eventrelated design.

We also found several activations in the left and medial frontal lobe for words compared to pseudowords. Activations in the left and medial frontal lobe have repeatedly been reported in neuroimaging studies investigating semantic processing (e.g., Roskies, Fiez, Balota, Raichle, \& Petersen, 2001; Wagner et al., 1998; Démonet et al., 1992). However, lesions in frontal areas have not been reported to cause semantic deficits. This suggests that frontal areas rather exert a control function with regard to the effortful retrieval of semantic information from posterior sources (e.g., Fiez, 1997). In the present study, however, there was little overlap with regard to the locus of activation for the two tasks. This difference might be ascribable to the higher degree of cognitive control and performance monitoring required in the case of the phonological lexical decision task, as compared to the silent articulation task, because medial frontal areas are assumed to play a major role with regard to these functions (MacDonald, Cohen, Stenger, \& Carter, 2000). 


\section{Conclusion}

In reading regular Japanese Kana orthography, the visual familiarity of words, which was found to facilitate reaction times in off-line reading and phonological lexical decision tasks, was found to give rise to less activation in the left inferior prefrontal gyrus, a brain area previously associated with phonological encoding. This is consistent with the dual-route model, which assumes a route of sequential phonological assembly for visually unfamiliar words and pseudowords, whereas the pronunciation of visually familiar words can be accessed directly via the visual word form lexicon. However, no evidence was found for a brain area that shows an increase in activation for visually highly familiar words. If, on the other hand, less activation is expected for visually familiar words in the visual word form area (search process), it is possible that one of the areas found more active for unfamiliar words compared to familiar words might also be involved in visual word form processing.

Compared to pseudowords, words were found to activate areas more strongly in the left and right temporo-parietal region, the middle part of the middle/ inferior temporal gyrus, and the posterior cingulate, independent of visual familiarity. These areas could be related to the processing of lexical status or the processing of meaning. The higher activation for words than for pseudowords, however, is difficult to reconcile with the idea of a resource demanding lexical search. It is therefore plausible that these areas are involved in auditory word form recognition (similar to the face recognition area) or in the processing of word meaning.

\section{METHODS}

\section{Participants}

Twenty-four students from the psychology department of the Nihon University ( 7 men, 17 women, between 18 to 22 years of age, mean age 20.1) took part in the two behavioral experiments (12 in reading aloud, 12 in phonological lexical decision). An additional 18 students from the psychology department of the Nihon University (8 men, 10 women, between 18 to 28 years of age, mean age 22.3) took part in the two experiments involving an fMRI measurement (10 in Experiment 1, 8 in Experiment 2). The data of one participant in Experiment 2 had to be discarded from the analysis due to scanner malfunction. All participants who underwent fMRI measurement were right-handed (Edinburgh Handedness Inventory) and had not taken part in any of the behavioral experiments. They were informed in detail of the procedure and had signed a written form of consent. All participants were native speakers of Japanese and were compensated for their participation.

\section{Materials}

The same materials were used in all four experiments. The two-factorial $(2 \times 3)$ design consisted of the factors type of Kana script (Hiragana, Katakana) and word type (familiar representation, unfamiliar representation, and pseudoword). Thirty-six experimental and two practice items were selected for each of the six conditions. The word stimuli were selected from the NTT database (Amano \& Kondo, 2000), such that they had a high rating for overall word familiarity and a very high (low) rating for their presentation in one of the two Kana scripts (script familiarity). The conditions Katakana familiar and Hiragana unfamiliar were realized by selecting 76 well-known foreign loanwords that had a high script familiarity rating for Katakana and a low rating for Hiragana. Two stimulus lists were prepared with one half of the words assigned randomly to each condition for one half of the participants. For the Hiragana familiar condition, 38 well-known words with a high script familiarity rating for Hiragana were used. The unfamiliar Katakana condition, however, was not realized by rewriting the familiar Hiragana words in Katakana. Visually familiar Hiragana words have a higher script variability than visually familiar Katakana words. Rewriting the Hiragana words with Katakana would therefore not render them visually very unfamiliar. The condition Katakana unfamiliar was therefore realized differently: 38 well-known words, which are usually written with Chinese characters, were selected because of their very low script familiarity rating for their appearance in Katakana. For example, the word for university, da.i.ga.$\mathrm{ku}$, is usually written with Chinese characters 大学 and judged as being visually very unfamiliar when presented in Katakana ダイガク (see, for a similar choice of stimuli, Besner \& Hildebrandt, 1987; Hatta et al., 1984). Additionally, 76 pseudowords were constructed by changing a syllable of existing Kana words and checked by three native speakers. Thirty-eight pseudowords were assigned randomly to the Katakana and Hiragana pseudoword conditions for one half of the participants. Word length varied from two to four Kana characters. The 216 experimental trials of the experiment were divided into six runs, each run consisting of 2 practice and 36 experimental trials. The trials were randomized separately for every run and every participant.

\section{Procedure}

\section{Behavioral Experiments}

First, a white fixation cross appeared for $500 \mathrm{msec}$ in the middle of a black screen followed after $750 \mathrm{msec}$ by the response-terminated presentation of a word or pseudoword. The following trial was presented 2000 msec after the response of the participant. Immediate feedback was given in the case of an error and a practice trial was inserted. Inserting a practice trial reduces the overall 
variance of reaction time data because the reaction on trials following an error has been observed to be considerably delayed (Rabbitt \& Rodgers, 1977). Participants received 10 practice trials at the beginning of the experiment and four at the start of each new run. In the first experiment, participants were instructed to read each stimulus aloud. Reaction times were measured by means of a voice key. After each trial the experimenter typed in a code indicating a correct response or the type of the error (errors made by the participant, and technical errors, such as premature or delayed voice-key triggering). In the second experiment, participants had to press one button on a button box connected to the computer controlling the experiment when the stimulus presented was a word or sounded like a word, and to press another button when it was a pseudoword (phonological lexical decision task).

\section{fMRI Experiments}

An event-related design was used for both fMRI experiments. Each run was randomized separately for each participant, subject to the following constraint: Each transition between the six conditions occurred exactly once for every run. This measure was taken to control sequential effects on the slow physiological response measured by fMRI (Dale \& Buckner, 1997). Each run began with the presentation of a white fixation cross on a black background in the middle of the screen. It was replaced by a single word or pseudoword stimulus, shown for $800 \mathrm{msec}$. The fixation cross then reappeared and remained on the screen until the presentation of the next stimulus. Stimuli were presented every $7350 \mathrm{msec}$ (ISI = $6550 \mathrm{msec}$ ). Every other stimulus was synchronized with the onset of the scanner cycle, while the other half of the stimuli was presented in the middle of a TR to ensure a more representative sample of the BOLD signal (Miezin, Maccotta, Ollinger, Petersen, \& Buckner, 2000). The word stimuli were projected onto a mirror at a comfortable viewing distance. A single Kana character subtended a visual angle of approximately $2.3^{\circ}$. Foam paddings were used to attenuate head movements and scanner noise. Between runs, participants were given a short rest of at least $30 \mathrm{sec}$. In Experiment 1, the participants were instructed to silently articulate each Kana character string carefully while avoiding head or jaw movements. In Experiment 2, participants had to perform a phonological lexical decision task. Word responses were given with the thumb, pseudoword responses with the index finger of the right hand. Button presses were recorded inside the scanner using a switch and an infrared-based interface.

\section{Image Acquisition}

All images were acquired with a 1.5-T Siemens Symphony MRI scanner. Functional images were obtained using a gradient-echo EPI sequence (20 axial slices in the ACPC plane, slice thickness $5 \mathrm{~mm}$, voxel $3 \times 3 \mathrm{~mm}$, gap $0.5 \mathrm{~mm}$, FOV $192 \mathrm{~mm}$, TR $2000 \mathrm{msec}$, TE $50 \mathrm{msec}$ ). Additionally, a T1 anatomical scan $\left(1 \mathrm{~mm}^{3}\right.$ voxel, FOV of $256 \mathrm{~mm}$, TR $11.08 \mathrm{msec}$, TE $4.3 \mathrm{msec}$ ) was obtained for each participant.

\section{Data Preprocessing and Analysis}

Preprocessing and data analysis were performed using SPM99 software. ${ }^{2}$ The first seven functional scans were discarded to allow for magnetic saturation. A total of 126 scans was acquired per run and participant. The individual slices of a functional volume (20 slices in total) were temporally corrected for their acquisition time difference with regard to the middle (tenth) slice. The functional images of each participant were realigned to a mean image to correct for head motion. Functional scans were coregistered to the anatomical scan and normalized to the MNI brain template. They were smoothed in the spatial (isotropic Gaussian kernel of $12 \mathrm{~mm}$ FWMH) and temporal domain (high pass, cutoff $1 / 88 \mathrm{~Hz}$ ). The delta function of the stimulus onsets for each condition was convolved with the canonical HRF and its first temporal derivative, $\mathrm{HRF}^{\prime}$, in order to account for minor latency differences (Friston et al., 1998). A fixed effects group analysis based on the general linear model was calculated, with six parameters (the contribution of the HRF for the six conditions) entered as predictors of interest. The estimates for the contribution of the $\mathrm{HRF}^{\prime}$, motion parameters, and a constant for global mean activation per session were included in the model as covariates of no interest. Linear contrasts for the differential main effects of the six conditions were calculated. The resultant statistical parameter map of $t$ values was thresholded at $p<.001$ uncorrected, reporting only cluster coordinates surpassing a $p$ value of .05, corrected at cluster level. In Experiment 2, (phonological lexical decision task) errors were discarded from the statistical analysis. For the joint analysis of both experiments a random effects analysis was computed using the same threshold as for the fixed effects analysis. The data in the ROI plots are given as normalized values (grand mean scaling) and have been preprocessed (filtering: HP, $0.01 \mathrm{~Hz}$; LP, $0.125 \mathrm{~Hz}$; linearly detrended).

\section{Acknowledgments}

The authors thank Wolfgang Schwarz and Herbert Schriefers for providing valuable comments on earlier versions of this manuscript. This work was supported by the Netherlands Organization for Scientific Research (NWO) grants No. R56-450 and No. R58-104 awarded to the first author, and a grant from the Ministry of Education, Culture, Sports, Science and Technology to promote multidisciplinary research projects, "Brain Mechanisms for Memory, Cognition, and Behavior" awarded to M. T. 
Reprint requests should be sent to Anja Ischebeck, Max Planck Institute of Cognitive Neuroscience, Stephanstrasse 1a, 04103 Leipzig, Germany, or via e-mail: ischebeck@cns.mpg.de.

The data reported in this experiment have been deposited in the fMRI Data Center (http://www.fmridc.org). The accession number is 2-2003-114EP.

\section{Notes}

1. Brodmann's areas are approximations derived from the Talairach and Tournoux brain atlas (1988).

2. A correction is applied to the coordinates given by SPM so that they conform more closely to the coordinate system of Talairach and Tournoux (1988): $x_{\mathrm{TT}}=0.99 x_{\mathrm{SPM}}$. If $z_{\mathrm{SPM}}<0$ : $y_{\mathrm{TT}}=0.9688 y_{\mathrm{SPM}}+0.042 z_{\mathrm{SPM}}$ and $z_{\mathrm{TT}}=-0.0485 y_{\mathrm{SPM}}+$ $0.839 z_{\mathrm{SPM}}$. If $z_{\mathrm{SPM}}>0: y_{\mathrm{TT}}=0.9688 y_{\mathrm{SPM}}+0.046 z_{\mathrm{SPM}}$ and $z_{\mathrm{TT}}=$ $-0.0485 y_{\mathrm{SPM}}+0.9189 z_{\mathrm{SPM}}$. This correction is applied throughout the present article for all given coordinates (Brett et al., 2002; www.mrc-cbu.cam.ac.uk/Imaging/common/mnispace. shtml).

\section{REFERENCES}

Amano, S., \& Kondo, T. (2000). Nibongo no Goi Tokusei [Lexical properties of Japanese]. NTT database series. Tokyo: Sanshodo.

Beauregard, M., Chertkow, H., Bub, D., Murtha, S., Dixon, R., \& Evans, A. (1997). The neural substrate for concrete, abstract, and emotional word lexica: A positron emission tomography study. Journal of Cognitive Neuroscience, 9, 441-461.

Besner, D., \& Hildebrandt, N. (1987). Orthographic and phonological codes in the oral reading of Japanese Kana. Journal of Experimental Psychology: Learning, Memory, and Cognition, 13, 335-343.

Besner, D., \& Smith, M. C. (1992). Basic processes in reading: Is the orthographic depth hypothesis sinking? In R. Frost \& L. Katz (Eds.), Orthography, Phonology, Morphology, and Meaning (pp. 45-66). Amsterdam: Elsevier.

Binder, J. R. (1999). Functional MRI of the language system. In C. T. W. Moonen \& P. A. Bandettini (Eds.), Functional MRI (pp. 407-419). Berlin: Springer.

Binder, J. R., Frost, J. A., Hammeke, T. A., Bellgowan, P. S. F., Rao, S. M., \& Cox, R. W. (1999). Conceptual processing during the conscious resting state: A functional fMRI study. Journal of Cognitive Neuroscience, 11, 80-95.

Binder, J. R., Frost, J. A., Hammeke, T. A., Cox, R. W., Rao, S. M., \& Prieto, T. (1997). Human language areas identified by functional magnetic resonance imaging. Journal of Neuroscience, 17, 353-362.

Binder, J. R., McKiernan, K. A., Parsons, M. E., Westbury, C. F., Possing, E. T., Kaufmann, J. N., \& Buchanan, L. (2003). Neural correlates of lexical access during visual word recognition. Journal of Cognitive Neuroscience, 15 , 372-393.

Brett, M., Johnsrude, I. S., \& Owen, A. M. (2002). The problem of functional location in the human brain. Nature Reviews Neuroscience, 3, 243-249.

Cohen, L., Dehaene, S., Naccache, L., Lehericy, S., DehaeneLambertz, G., Henaff, M.-A., \& Michell, F. (2000). The visual word form area. Spatial and temporal characterization of an initial stage of reading in normal subjects and posterior splitbrain patients. Brain, 123, 291-307.

Coltheart, M., Curtis, B., Atkins, P., \& Haller, M. (1993). Models of reading aloud: Dual-route and parallel-distributed processing approaches. Psychological Review, 100, 589-608.
Dale A. M., \& Buckner, R. L. (1997). Selective averaging of rapidly presented individual trials using fMRI. Human Brain Mapping, 5, 329-340.

Damasio, A. R., \& Damasio, H. (1983). The anatomic basis of pure alexia. Neurology, 33, 1573-1583.

Dehaene, S., Naccache, L., Cohen, L, Le Bihan, D., Mangin, J.-F., Poline, J.-B., \& Rivière, D. (2001). Cerebral mechanisms of word masking and unconscious repetition priming. Nature Neuroscience, 4, 752-758.

Démonet, J. F., Chollet, F., Ramsay, S., Cardebat, D., Nespoulous, J. L., Wise, R., Rascol, A., \& Frackowiak, R. (1992). The anatomy of phonological and semantic processing in normal subjects. Brain, 115, 1753-1768.

d'Esposito, M. D., Zarahn, E., \& Aguirre, G. K. (1999). Event-related functional MRI: Implications for cognitive psychology. Psychological Bulletin, 125, 155-164.

Donaldson, D. I., \& Buckner, R. L. (2001). Effective paradigm design. In P. Jezzard, P. M. Matthews, \& S. M. Smith (Eds.), Functional MRI: An Introduction to Methods (pp. 177-195). New York: Oxford University Press.

Dong, Y., Fukuyama, H., Honda, M., Okada, T., Hanakawa, T., Nakamura, K., Nagahama, Y., Nagamine, T., Konishi, J., \& Shibasaki, H. (2000). Essential role of the right superior parietal cortex in Japanese Kana mirror reading. An fMRI study. Brain, 123, 790-799.

Epstein, R., \& Kanwisher, N. (1998). A cortical representation of the local visual environment. Nature, 392, 598-601.

Fiebach, C. J., Friederici, A. D., Müller, K., \& von Cramon, D. Y. (2002). fMRI evidence for dual routes to the mental lexicon in visual word recognition. Journal of Cognitive Neuroscience, 14, 11-23.

Fiez, J. A. (1997). Phonology, semantics and the role of the left inferior prefrontal cortex. Human Brain Mapping, 5, 79-83.

Fiez, J. A., Balota, D. A., Raichle, M. E., \& Petersen, S. E. (1999). Effects of lexicality, frequency, and spelling-to-sound consistency on the functional anatomy of reading. Neuron, 24, 205-218.

Forster, K. I. (1976). Accessing the mental lexicon. In R. J. Wales \& E. C. T. Walker (Eds.), New approaches to language mechanisms (pp. 257-287). Amsterdam: North-Holland.

Friston, K. J., Fletcher, P., Josephs, O., Holmes, A., Rugg, M. D., \& Turner, R. (1998). Event-related fMRI: Characterising differential responses. Neuroimage, 7, 30-40.

Friston, K. J., Price, C. J., Fletcher, P., Moore, C., Frackowiak, R. S. J., \& Dolan, R. J. (1996). The trouble with cognitive subtraction. Neuroimage, 4, 97-104.

Hagoort, P., Indefrey, P., Brown, C., Herzog, H., Steinmetz, H., \& Seitz, R. (1999). The neural circuitry involved in the reading of German words and pseudowords: A PET study. Journal of Cognitive Neuroscience, 11, 383-398.

Hatta, T., Katoh, H., \& Kirsner, K. (1984). Lexical representation of foreign loan words in Japanese learners among native speakers of English. Psychologia, 27, 237-243.

Herbster, A. N., Mintun, M. A., Nebes, R. D., \& Becker, J. T. (1997). Regional cerebral blood flow during word and non-word reading. Human Brain Mapping, 5, 84-92.

Howard, D., Patterson, K., Wise, R., Brown, W. D., Friston, K., Weiller, C., \& Frackowiak, R. (1992). The cortical localization of the lexicons. Brain, 115, 1769-1782.

Indefrey, P., Kleinschmidt, A., Merboldt, K.-D., Krüger, G., Brown, C., Hagoort, P., \& Frahm, J. (1997). Equivalent responses to lexical and non-lexical stimuli in occipital cortex: A functional magnetic resonance imaging study. Neuroimage, 5, 78-81.

Iwata, M. (1984). Kanji vs. Kana. Neuropsychological correlates of the Japanese writing system. Trends in Neurosciences, 7, 290-293. 
Kanwisher, N., McDermott, J., \& Chun, M. M. (1997). The fusiform face area: A module in human extrastriate cortex specialized for face perception. Journal of Neuroscience, 17, 4302-4311.

Katz, L., \& Frost, R. (1992). The reading process is different for different orthographies: The orthographic depth hypothesis. In R. Frost \& L. Katz (Eds.), Orthography, phonology, morphology, and meaning (pp. 67-84). Amsterdam: Elsevier.

Kawakami, M. (1993). Kana-go no goi kettei kadai ni okeru hyôki no shinkinsei to shori tan'i [Script familiarity and processing unit in lexical decision with Japanese Kana words]. Japanese Journal of Psychology, 64, 235-239.

Law, I., Kannao, I., Fujita, H., Lassen, N. A., Miura, S., \& Uemura, K. (1991). Left supramarginal/angular gyri activation during reading of syllabograms in the Japanese language. Journal of Neurolinguistics, 6, 243-251.

MacDonald, A. W., Cohen, J. D., Stenger, V. A., \& Carter, C. S. (2000). Dissociating the role of the dorsolateral prefrontal and anterior cingulate cortex in cognitive control. Science, 288, 1835-1838.

Mayall, K., Humphreys, G. W., Mechelli, A., Olson, A., \& Price, C. J. (2001). The effects of case mixing on word recognition: Evidence from a PET study. Journal of Cognitive Neuroscience, 13, 844-853.

Menard, M. T., Kosslyn, S. M., Thompson, W. L., Alpert, N. M., \& Rauch, S. L. (1996). Encoding words and pictures: A positron emission tomography study. Neuropsychologia, 34, 185-194.

Miezin, F. M., Maccotta, L., Ollinger, J. M., Petersen, S. E., \& Buckner, R. L. (2000). Characterizing the hemodynamic response: Effects of presentation rate, sampling procedure, and the possibility of ordering brain activity based on relative timing. Neuroimage, 11, 735-759.

Mishkin, M., Ungerleider, L. G., \& Macko, K. A. (1983). Object vision and spatial vision: Two cortical pathways. Trends in Neurosciences, 6, 414-417.

Paulesu, E., McCrory, E., Fazio, F., Menoncello, L., Brunswick, N., Cappa, S. F., Cotelli, M., Cossu, G., Corte, F., Lorusso, M., Pesenti, S., Gallagher, A., Perani, D., Price, C., Frith, C. D., \& Frith, U. (2000). A cultural effect on brain function. Nature Neuroscience, 3, 91-96.

Petersen, S. E., Fox, P. T., Posner, M. I., Mintun, M., \& Raichle, M. E. (1988). Positron emission tomographic studies of cortical anatomy of single words processing. Nature, 331, 585-589.

Petersen, S. E., Fox, P. T., Snyder, A. Z., \& Raichle, M. E. (1990). Activation of extrastriate and frontal cortical areas by visual words and word-like stimuli. Science, 249, 1041-1044.

Polk, T. A., \& Farah, M. J. (2002). Functional MRI evidence for an abstract, not visual, word-form area. Journal of Experimental Psychology: General, 131, 65-72.

Polk, T. A., Stallcup, M., Aguirre, G. K., Alsop, D. C., D'Esposito, M., Detre, J. A., \& Farah, M. J. (2002). Neural specialization for letter recognition. Journal of Cognitive Neuroscience, 14, 145-159.

Price, C. J. (2000). The anatomy of language: Contributions from functional neuroimaging. Journal of Anatomy, 197, 335-359.

Price, C. J., Indefrey, P., \& van Turennout, M. (1999). The neural architecture underlying the processing of written and spoken word forms. In C. M. Brown \& P. Hagoort (Eds.), The neurocognition of language (pp. 211-240). New York: Oxford University Press.

Pugh, K. R., Shaywitz, B. A., Shaywitz, S. E., Constable, R. T., Skudlarski, P., Fulbright, R. K., Bronen, R. A., Shankweiler, D. P., Katz, L., Fletcher, J. M., \& Gore, J. C. (1996). Cerebral organization of component processes in reading. Brain, 119, 1221-1238.

Rabbitt, P., \& Rodgers, B. (1977). What does a man do after he makes an error? An analysis of response programming. Quarterly Journal of Experimental Psychology, 29, 727-743.

Roskies, A. L., Fiez, J. A., Balota, D. A., Raichle, M. E., \& Petersen, S. E. (2001). Task-dependent modulation of regions in the left inferior frontal cortex during semantic processing. Journal of Cognitive Neuroscience, 13, 829-843.

Rumsey, J. M., Horwitz, B., Donohue, B. C., Nace, K., Maisog, J. M., \& Andreason, P. (1997). Phonological and orthographic components of word recognition. A PET-rCBF study. Brain, 120, 739-759.

Tagamets, M.-A., Novick, J. M., Chalmers, M. L., \& Friedman, R. B. (2000). A parametric approach to orthographic processing in the brain: An fMRI study. Journal of Cognitive Neuroscience, 12, 281-297.

Talairach, J., \& Tournoux, P. (1988). Co-planar stereotactic atlas of the buman brain. New York: Thieme.

Taylor, T. E., \& Lupker, S. J. (2001). Sequential effects in naming: A time-criterion account. Journal of Experimental Psychology: Learning, Memory, and Cognition, 27, 117-138.

Usui, N. (1998). Kana tango no ninchi ni okeru zentaiteki shori no kentô. [On the role of holistic processing in the recognition of Japanese Kana words]. Japanese Journal of Psychology, 69, 105-112.

Vance, T. J. (1987). An introduction to Japanese phonology. Albany: State University of New York Press.

Wagner, A. D., Schacter, D. L., Rotte, M., Koutstaal, W., Maril, A., \& Dale, A. M. (1998). Science, 281, 1188-1191.

Xu, B., Grafman, J., Gaillard, W. D., Ishii, K., Vega-Bermudez, F., Pietrini, P., Reeves-Tyler, P., DiCamillo, P., \& Theodore, W. (2001). Conjoint and extended neural networks for the computation of speech codes: The neural basis of selective impairment in reading words and pseudowords. Cerebral Cortex, 11, 267-277.

Yamada, J., Imai, H., \& Ikebe, Y. (1990). The use of the orthographic lexicon in reading. Journal of General Psychology, 117, 311-323.

Yamadori, A. (2000). Neuropsychological model of reading based on Japanese experiences. Psychologia, 43, 1-14. 